\title{
CALIDAD DE UN MOOC SOBRE INCLUSIÓN EDUCATIVA: APLICACIÓN DE VARIOS INSTRUMENTOS E INDICADORES
}

\author{
Patricia GÓMEZ HERNÁNDEZ ${ }^{1}$ \\ Carlos MONGE LÓPEZ ${ }^{2}$ \\ Eladio SEBASTIÁN HEREDERO ${ }^{3}$
}

Resumo: En pleno siglo XXI parece significativa la necesidad de la formación en, para y con las Tecnologías de la Información y Comunicación y la atención a la diversidad. En este marco contextual pueden destacarse dos elementos fundamentales: los Massive Open Online Courses y la escuela inclusiva. Así, el objetivo principal de este estudio es evaluar un $M O O C$ del contexto iberoamericano que enfatice la atención a la diversidad desde una perspectiva de educación inclusiva. Para ello se empleó el estudio de caso como método de investigación, destacando la aplicación de varios instrumentos de evaluación en relación a criterios de calidad de MOOCs. De manera general, los resultados destacan una valoración muy positiva en casi todas las dimensiones y subdimensiones analizadas, especialmente en los aspectos más pedagógicos. A modo de conclusión, se puede señalar la existencia de un $M O O C$ en el contexto iberoamericano que aborda la educación inclusiva, el cual ha sido valorado muy positivamente, aunque también debe seguir mejorando.

Palavras-chave: Calidad. Inclusión. Evaluación. COMA.

\section{INTRODUCCIÓN}

Dada la diversidad cada vez más prominente en el siglo XXI, momento también de pleno avance de las Tecnologías de la Información y Comunicación, el sistema educativo debe afrontar ciertos retos indiscutibles y propiciar el pleno desarrollo de la comunidad. Así, la escuela ha de posicionarse en el marco de una escuela inclusiva, entendida como un proceso basado en la participación de toda la comunidad, y especialmente de aquellos grupos o individuos en riesgo de exclusión, modificando culturas, políticas y prácticas, con el fin de potenciar el máximo desarrollo en el ámbito personal y social (BOOTH; AINSCOW, 2011).

\footnotetext{
1 Departamento de Ciencias de la Educación. UAH - Universidad de Alcalá. Alcalá de Henares Comunidad de Madrid - España. 28801 - patricia.gomezh@uah.es.

${ }^{2}$ Departamento de Ciencias de la Educación. UAH - Universidad de Alcalá. Facultad de Educación. Guadalajara - Castilla-La Mancha - España. 19001 - carlos.monge@uah.es.

${ }^{3}$ Departamento de Ciencias de la Educación. UAH - Universidad de Alcalá. Facultad de Educación. Guadalajara - Castilla-La Mancha - España. 19001 - eladio.sebastian@uah.es.
} 
Otro de los fenómenos que se produce en este contexto tecnológico es el surgimiento y apogeo de los Massive Open Online Courses (MOOCs), facilitando el acceso a la formación a una cantidad innumerable de personas en diversos contextos económicos, laborales, geográficos, etc. Este recurso se puede considerar como cursos en línea en los que no existe ningún requisito para acceder a ellos y que están preparados para aceptar a miles de estudiantes participando de forma simultánea (LUJÁN, 2013). A pesar de estar avalados la mayoría de ellos por universidades de reconocido prestigio a nivel internacional, la creciente expansión de MOOCs conlleva asociadas ciertas dudas sobre la calidad que presentan. Como apuntan Cabero, Llorente y Vázquez (2014), algunos autores creen que estos cursos son una aplicación tecnológica educativa que facilita y garantiza una formación de calidad para todas las personas, mientras que otros autores creen que los MOOCs suponen simplemente un nuevo modelo de negocio para las instituciones de educación superior, dejando mucho que desear en cuestiones de calidad. En este sentido, Roig, Mengual y Suárez (2014) analizan 52 MOOCs de 10 plataformas distintas, hallando que casi todas las dimensiones evaluadas en relación a la calidad pedagógica de estos cursos se aproximan a la media de la escala empleada (metodología, organización, motivación, multimedia, lenguaje, valores, singularidad) y considerando el $67.40 \%$ de los cursos con media calidad y el $13.20 \%$ con alta calidad, además de destacar que la existencia de una guía didáctica, de objetivos explícitamente planteados y de actividades de refuerzo contribuye a mejorar sustancialmente la calidad de los MOOCs. Por su parte, Walls et al. (2015) proponen un modelo para asegurar la calidad de estos cursos, que se extiende a: (a) la calidad de desarrollo, (b) la calidad de la administración y de los estudiantes, (c) la calidad de los resultados de aprendizaje y (d) la calidad de los resultados institucionales. Efectivamente, los modelos e instrumentos para evaluar la calidad de estos cursos resultan muy diversos (AGUADED; MEDINA, 2015; ALEMÁN; SANCHO; GÓMEZ, 2015; GEA, 2015; MENGUAL; LLORET; ROIG, 2015; RAMÍREZ, 2015; RAMÍREZ; SALMERÓN, 2015; RAMÍREZ; SALMERÓN; LÓPEZ, 2015; ROIG; MENGUAL; SUÁREZ, 2014), sin alcanzar un claro consenso de criterios, indicadores y procedimientos, al menos en el contexto iberoamericano. Así, Ramírez Salmerón y López (2015) idean un modelo de evaluación según ADECUR y las Normas UNE66181:2012 (RAMÍREZ; SALMERÓN; LÓPEZ, 2015) con los indicadores comunes y no comunes: 
- Indicadores comunes de calidad: dimensión de metodología de aprendizaje (diseño didáctico-instruccional; recursos formativos y actividades de aprendizaje; tutoría; entorno tecnológico digital de aprendizaje).

- Indicadores de las Normas UNE66181:2012: dimensión de reconocimiento de la formación para la empleabilidad (reconocimiento de la formación para la empleabilidad), dimensión de metodología de aprendizaje (diseño didácticoinstruccional; tutoría; entorno tecnológico de aprendizaje) y dimensión de niveles de accesibilidad (accesibilidad hardware; accesibilidad software; accesibilidad web).

- Indicadores de ADECUR: dimensión psicodidáctica (ambiente virtual; aprendizaje; contenidos; accesibilidad y secuenciación; evaluación y acción; tutorial) y dimensión técnico-estética (recursos y aspectos técnicos).

De igual manera, en una profunda revisión, Aguaded y Medina (2015) señalan varias clasificaciones de la calidad en los MOOCs:

- $\quad$ Autonomía, diversidad, apertura e interactividad.

- $\quad$ Público objetivo, métodos de enseñanza-aprendizaje, explicaciones, resultados de aprendizaje, teorías subyacentes de aprendizaje y estructura del curso.

- Guía didáctica, metodología, organización de los contenidos, calidad de los contenidos, recursos didácticos, capacidad de motivación, elementos multimedia, estilo del lenguaje, valores y singularidad de los usuarios.

- Planificación, programa, recursos, desarrollo del proceso y calidad de los resultados.

- Uso de las herramientas tecnológicas y planificación de los componentes curriculares.

Como apuntan García Barrera, Gómez Hernández y Monge López (En prensa) en su propuesta metodológica, es indudable la relación existente entre los MOOCs y la atención a la diversidad en el ámbito educativo, pues estos cursos brindan grandes oportunidades para la inclusión educativa, aunque todavía queda mucho por mejorar.

Por todo ello, el objetivo principal de este estudio es ofrecer un análisis de un $M O O C$ sobre educación inclusiva en el contexto iberoamericano mediante la aplicación de distintos instrumentos de evaluación.

\section{Método}

Se trata de un estudio de caso de tipo exploratorio, evaluativo, único e intrínseco, lo que posibilita una fuerte validez de los datos, un reconocimiento de la realidad social, reinterpretaciones, un paso para la acción, mayor accesibilidad, etc. (COHEN; MANION; MORRISON, 2007).

El caso seleccionado es el MOOC titulado "Pedagogía hospitalaria". Este curso es el único de MiríadaX (la plataforma más importante en Iberoamérica) que responde a las palabras “inclusión”/“inclusiva” y “diversidad” en el área de Pedagogía. 
Sobre esta base se diseña este curso cuyos objetivos son dos: en primer lugar, analizar el impacto de la enfermedad, y el hecho añadido del ingreso hospitalario, sobre la vida personal y social tanto del niño o el adolescente, como de su familia; y en segundo lugar, presentar diversas propuestas de actuación desde la Pedagogía Hospitalaria para la inclusión social y escolar del alumno en situación de enfermedad. (ESPAÑA, 2015).

Diseñado desde la Universidad de Navarra, se planifica en siete semanas de trabajo (17.5 horas de estudio estimadas) para un nivel superior al introductorio (conocimientos necesarios: nociones básicas de Pedagogía). Se estructura en siete módulos de aprendizaje:

- $\quad$ Módulo 0. Presentación.

- Módulo 1. Contextualizando la pedagogía hospitalaria.

- Módulo 2. La enfermedad en la etapa infanto-juvenil.

- Módulo 3. La hospitalización y sus características.

- Módulo 4. Pautas generales de actuación pedagógica en el hospital.

- Módulo 5. Las aulas hospitalarias.

- Módulo 6. Atención educativa domiciliaria.

- Módulo 7. La vuelta al colegio.

La recogida de datos se basa en distintos cuestionarios sobre la calidad de los MOOCs. El cuestionario empleado por Alemán, Sancho y Gómez (2015) consta de preguntas cerradas que valoran 50 indicadores con una escala tipo Likert de 4 puntos, clasificándose los indicadores en 15 subcategorías relacionadas con los factores pedagógicos (contenidos, enfoque pedagógico, tutorial-evaluación, adecuación/adaptación a los usuarios, capacidad de motivación y recursos), factores funcionales (facilidad de uso, autonomía-control del usuario y funcionalidad de la documentación), factores tecnológicos (interacción/diálogo, navegación, entorno visual, diseño tecnológico y versatilidad) y factores temporales (presentación de exámenes, realización de actividades, realización de ejercicios, estudio de temas, calendario/agenda y participación en los foros de discusión). El Cuestionario de evaluación de la calidad de cursos virtuales adaptado a MOOCs (MENGUAL et al., 2015) consta de tres dimensiones con ítems de tipo Likert (1-5 puntos): (1) calidad en la comunicación y elementos multimedia (12 ítems), (2) coherencia curricular y adaptación al usuario (9 ítems) y (3) planificación didáctica (11 ítems). EduTool (RAMÍREZ, 2015; RAMÍREZ; SALMERÓN, 2015) es una herramienta para la evaluación de la calidad de los MOOCs, pero por su compleja aplicación para este estudio se simplifica según las 
subdimensiones: (1.1) reconocimiento de la formación para la empleabilidad, (2.1) diseño didáctico-instruccional, (2.2) recursos formativos y actividades de aprendizaje, (2.3) tutoría, (2.4) entorno tecnológico-digital de aprendizaje, (3.1) accesibilidad de hardware, (3.2) accesibilidad de software y (3.3) accesibilidad web. La propuesta de indicadores para uMuMooc (GEA, 2015; GUERRERO, 2015) recoge tres dimensiones: (1) planificación/gestión (administración/gestión y acreditación/certificación), (2) diseño de aprendizaje (diseño didáctico-instruccional; contenidos; recursos y actividades; evaluación) y (3) comunicación-interacción (comunicación y tutorías).

\section{Resultados}

De forma general, se puede afirmar que este $M O O C$ es valorado positivamente en todas las dimensiones independientemente del instrumento empleado (Tabla 1). Así, siguiendo las ideas de Alemán, Sancho e Gómez (2015), pese a necesitar una revisión de la presentación de los exámenes (obteniendo una puntuación de 2 sobre 4), se obtiene una máxima puntuación en: contenidos; enfoque pedagógico; tutorial y evaluación; facilidad de uso; autonomía y control del usuario; navegación; entorno visual; diseño y tecnología; versatilidad; realización de actividades; realización de ejercicios; calendario/agenda; participación en foros de discusión. Por otro lado, dentro del Cuestionario de evaluación de la calidad de cursos virtuales adaptado a MOOCs (MENGUAL; LLORET; ROIG, 2015), se presenta una tendencia ligeramente más baja que en los resultados del instrumento anterior, obteniendo una puntuación alta (de 4.33 sobre 5) en calidad en la comunicación y elementos multimedia, ligeramente más baja en coherencia curricular y adaptación al usuario (4 puntos) y una puntuación de 3.64 en planificación didáctica. Uno de los instrumentos que más destaca dentro de este estudio, debido a la diferencia de puntuaciones encontrada entre las categorías estudiadas, es EduTool (RAMÍREZ, 2015; RAMÍREZ; SALMERÓN, 2015), donde el MOOC analizado presenta una puntuación baja (1 de 5 puntos) en reconocimiento de la formación para el empleo, media (3 puntos sobre 5) para tutoría, accesibilidad de hardware, de software y de web y una puntuación alta (5 puntos) para el entorno tecnológico-digital de aprendizaje. Y, por último, en la herramienta de evaluación uMuMooC (GEA, 2015; GUERRERO, 2015) se consigue una puntuación relativamente alta en prácticamente todas las subcategorías analizadas, destacando las tutorías, con la puntuación más baja (3.5 puntos sobre 5), y la administración/gestión y la comunicación como las categorías con la máxima puntuación (5 puntos). 
Tabla 1 - Resultados en la aplicación de varios instrumentos

\begin{tabular}{|c|c|c|}
\hline \multicolumn{2}{|c|}{ Cuestionario de calidad de MOOCs (ALEMÁN et al., 2015) } & Evaluación (1-4) \\
\hline \multirow[t]{6}{*}{ Categoría pedagógica } & Contenidos & 4 \\
\hline & Enfoque pedagógico & 4 \\
\hline & Tutorial y evaluación & 4 \\
\hline & $\begin{array}{l}\text { Adecuación y adaptación a los } \\
\text { usuarios }\end{array}$ & 3 \\
\hline & Capacidad de motivación & 3 \\
\hline & Recursos & 3 \\
\hline \multirow[t]{3}{*}{ Categoría funcional } & Facilidad de uso & 4 \\
\hline & Autonomía y control del usuario & 4 \\
\hline & Funcionalidad de la documentación & 3 \\
\hline \multirow[t]{5}{*}{ Categoría tecnológica } & Interacción y diálogos & 3 \\
\hline & Navegación & 4 \\
\hline & Entorno visual & 4 \\
\hline & Diseño y tecnología & 4 \\
\hline & Versatilidad & 4 \\
\hline \multirow[t]{6}{*}{ Categoría temporal } & Presentación de los exámenes & 2 \\
\hline & Realización de actividades & 4 \\
\hline & Realización de ejercicios & 4 \\
\hline & Estudio de temas & 3 \\
\hline & Calendario/agenda & 4 \\
\hline & Participación en foros de discusión & 4 \\
\hline \multicolumn{2}{|c|}{$\begin{array}{l}\text { Cuestionario de evaluación de la calidad de cursos virtuales adaptado a } \\
\text { MOOCs (MENGUAL; LLORET; ROIG, 2015) }\end{array}$} & Evaluación (1-5) \\
\hline \multicolumn{2}{|c|}{$\begin{array}{l}\text { Calidad en la comunicación y elementos multimedia } \\
\text { Coherencia curricular y adaptación al usuario }\end{array}$} & 4.33 \\
\hline \multicolumn{2}{|c|}{ Coherencia curricular y adaptación al usuario } & 4 \\
\hline \multicolumn{2}{|c|}{ Planificación didáctica } & 3.64 \\
\hline \multicolumn{2}{|c|}{ EduTool (RAMÍREZ, 2015; RAMÍREZ; SALMERÓN, 2015) } & Evaluación (1-5) \\
\hline \multicolumn{2}{|c|}{$\begin{array}{llll}\begin{array}{l}\text { Reconocimiento de } \\
\text { formación para el empleo }\end{array} & \text { la } & \begin{array}{l}\text { Reconocimiento de la formación } \\
\text { para el empleo }\end{array} & \\
\end{array}$} & 1 \\
\hline \multirow[t]{4}{*}{ Metodología de aprendizaje } & Diseño didáctico-instruccional & 4 \\
\hline & $\begin{array}{l}\text { Recursos formativos y actividades } \\
\text { de aprendizaje }\end{array}$ & 4 \\
\hline & Tutoría & 3 \\
\hline & $\begin{array}{l}\text { Entorno tecnológico-digital de } \\
\text { aprendizaje }\end{array}$ & 5 \\
\hline \multirow[t]{3}{*}{ Niveles de accesibilidad } & Accesibilidad de hardware & 3 \\
\hline & Accesibilidad de software & 3 \\
\hline & Accesibilidad web & 3 \\
\hline \multicolumn{2}{|c|}{ uMuMooC (GEA, 2015; GUERRERO, 2015) } & Evaluación (1-5) \\
\hline \multirow[t]{2}{*}{ Planificación/gestión } & Administración/gestión & 5 \\
\hline & Acreditación/certificación & 4 \\
\hline \multirow[t]{4}{*}{ Diseño de aprendizaje } & Diseño didáctico-instruccional & 4.29 \\
\hline & Contenidos & 4.50 \\
\hline & Recursos y actividades & 4.33 \\
\hline & Evaluación & 4.67 \\
\hline \multirow[t]{2}{*}{ Comunicación-interacción } & Comunicación & 5 \\
\hline & Tutorías & 3.5 \\
\hline
\end{tabular}

Fuente: Elaboración propia. 


\title{
Discusión y conclusiones
}

En líneas generales, se puede destacar la existencia de un único $M O O C$ en la plataforma más destacada del contexto iberoamericano que aborde el tema de la educación inclusiva. No obstante, este curso ha sido valorado muy positivamente en casi todas las dimensiones y subdimensiones evaluadas, independientemente del instrumento utilizado (ALEMÁN; SANCHO; GÓMEZ, 2015; GEA, 2015; GUERRERO, 2015; MENGUAL; LLORET; ROIG, 2015; RAMÍREZ, 2015; RAMÍREZ; SALMERÓN, 2015), aunque todavía quedan algunos aspectos que seguir mejorando.

Como futuras líneas de investigación, y dada la escasa existencia de MOOCs que enfatizan la educación desde una perspectiva inclusiva en Iberoamérica, se podría seguir trabajando con el estudio de caso de tipo investigación-acción, dando un paso más para la mejora de los procesos de enseñanza-aprendizaje (COHEN; MANION; MORRISON, 2007).

\section{QUALITY OF A MOOC INCLUSION ON EDUCATION: IMPLEMENTATION OF VARIOUS INSTRUMENTS AND INDICATORS}

\begin{abstract}
In the XXI century, the need of training in, for and with Information and Communication Technologies seems significant. In this contextual framework, there are two highlighted elements: the Massive Open Online Courses, and the inclusive school. Thus, the main aim of this study is to evaluate a MOOC from Iberoamerican context that emphasizes the attention to diversity from an approach based on inclusive education. For this reason, the case study was used as research method, standing out the application of several assessment instruments in relation to quality criteria of MOOCs. In general, the results highlight a positive evaluation around almost all analyzed dimensions and subdimensions, particularly around pedagogical aspects. In conclusion, there is a MOOC in the Iberoamerican context that discusses the inclusive education, which has been valued very positively, although it must continue to improve.
\end{abstract}

Key words: Assessment. Inclusion. MOOC. Quality.

\section{REFERÊNCIAS}

AGUADED, I.; MEDINA, R. Criterios de calidad para la valoración y gestión de MOOC. Revista Iberoamericana de Educación a Distancia, Loja, v.18, n.2, p.119$143,2015$.

ALEMÁN, L. Y.; SANCHO, T.; GÓMEZ, M. G. Indicadores de calidad pedagógica para el diseño de un curso en línea masivo y abierto de actualización docente. Revista de Universidad y Sociedad del Conocimiento, Barcelona, v.12, n.1, p.104-119, 2015. 
BOOTH, T.; AINSCOW, M. Index for inclusion. Developing learning and participation in schools. Bristol: Centre for Studies on Inclusive Education, 2011.

CABERO, J.; LLORENTE, M. C.; VÁZQUEZ, A. I. Las tipologías de MOOC: su diseño e implicaciones educativas. Profesorado. Revista de Currículum y Formación del Profesorado, Granada, v.18, n.1, p.13-26, 2014.

COHEN, L.; MANION, L.; MORRISON, K. Research methods in education. 6.ed. Nueva York: Routledge, 2007.

ESPAÑA. Pedagogía hospitalaria (MiríadaX). Navarra: Universidad de Navarra.

Disponible en: <https://www.mooc-list.com/course/pedagog\%C3\%ADa-hospitalariamir\%C3\%ADada-x?static=true >. Acceso en: 07 abr. 2016.

GARCÍA BARRERA, A.; GÓMEZ HERNÁNDEZ, P.; MONGE, C. La atención a la diversidad en los MOOC: una propuesta metodológica. Educación XX1, Madrid. (En prensa).

GEA, M. (Coord.). MOOC y criterios de calidad. Madrid: CRUE, 2015.

GUERRERO, C. uMuMooC: una propuesta de indicadores de calidad pedagógica para la realización de cursos MOOC. In: RUIZ, M. A. (Coord.). Educar para transformar: aprendizaje experiencial. Madrid: Universidad Europea de Madrid, 2015. p.231-236.

LUJÁN, S. Lecciones aprendidas en la organización de un curso de tipo MOOC. In: FIDALGO, Á; SEIN-ECHALUCE, M. L. (Ed.). II CONGRESO INTERNACIONAL SOBRE APRENDIZAJE, INNOVACIÓN Y COMPETITIVIDAD, 2013, Madrid. Anais... Madrid: Universidad Politécnica de Madrid, 2013.

MENGUAL, S.; LLORET, C.; ROIG, R. Validación del cuestionario de evaluación de la calidad de cursos virtuales adaptado a MOOC. Revista Iberoamericana de Educación a Distancia, Loja, v.18, n.2, p.145-169, 2015.

RAMÍREZ, M. B. La valoración de MOOC: una perspectiva de calidad. Revista Iberoamericana de Educación a Distancia, Loja, v.18, n.2, p.171-195, 2015.

.; SALMERÓN, J. L. EduTool: un instrumento para la evaluación y acreditación de la calidad de los MOOCs. Educación XX1, Madrid, v.18, n.2, p.97-123, 2015.

; LÓPEZ, E. Comparativa entre instrumentos de evaluación de calidad de cursos MOOC: ADECUR y Normas UNE 66181:2012. Revista de Universidad y Sociedad del Conocimiento, Barcelona, v.12, n.1, p.131-145, 2015.

ROIG, R.; MENGUAL, S.; SUÁREZ, C. Evaluación de la calidad pedagógica de los MOOC. Profesorado. Revista de Currículum y Formación del Profesorado, Granada, v.18, n.1, p.27-41, 2014.

WALLS, J. et al. Quality assurance for Massive Open Access Online Courses: building on the old to create something new. In: MCKAY, E.; LENARCIC, J. (Ed.). Macrolevel learning through Massive Open Online Courses (MOOCs). Strategies and predictions for the Future. Hershey: IGI-Global, 2015. p.25-47. 